\title{
Attenuated total reflection spectra of aqueous glycine in the terahertz region
}

\author{
Yuichi Ogawa $^{1 \mathrm{a})}$, Li Cheng ${ }^{1}$, Shin'ichiro Hayashi ${ }^{1,2}$, and \\ Kaori Fukunaga ${ }^{3}$ \\ ${ }^{1}$ Tohoku University, 1-1, Tsutsumidori Amamiya-machi Aoba-ku, Sendai, Miyagi \\ 981-8555, Japan \\ ${ }^{2}$ RIKEN SENDAI, 519-1399 Aramakiaoba, Aoba-ku, Sendai, Miyagi 980-0845, \\ Japan \\ ${ }^{3}$ National Institute of Information and Communications Technology, 4-2-1, \\ Nukui-Kitamachi, Koganei, Tokyo 184-8795, Japan \\ a)yogawa@bios.tohoku.ac.jp
}

\begin{abstract}
Attenuated total reflection (ATR) spectroscopy in the terahertz $(\mathrm{THz})$ region was used to measure the spectra of water, aqueous glycine solution, and glycine powder between 0.5 and $12 \mathrm{THz}$. By subtracting the absorption spectrum of water, we obtained clear absorption peaks for dissolved glycine at 2.1, 5.2, and $9.3 \mathrm{THz}$. In addition, we observed that the frequencies of these absorption peaks differed from the peaks for glycine powder. This indicates that the low-frequency vibrational mode in glycine molecules changes with the hydrogen bonding of glycine to $\mathrm{H}_{2} \mathrm{O}$ molecules.
\end{abstract}

Keywords: terahertz spectroscopy, attenuated total reflection, glycne aqueous solution

Classification: Science and engineering for electronics

\section{References}

[1] K. Kawase, Y. Ogawa, Y. Watanabe, and H. Inoue, "Non destructive terahertz imaging of illicit drugs using spectral fingerprints," Optics Express, vol. 11, no. 20, pp. 2549-2554, 2003.

[2] K. Fukunaga, Y. Ogawa, S. Hayashi, and I. Hosako, "Terahertz spectroscopy for art conservation," IEICE Electron. Express, vol. 4, no. 8, pp. 258-263, 2007.

[3] M. Tonouchi, "Cutting-edge terahertz technology," Nature Photonics, vol. 1, pp. 97-105, 2007.

[4] M. R. Querry, D. M. Wieliczka, and D. J. Segelstein, "Water (H2O)," in Handbook of Optical Constants of Solids II, E. D. Palik, ed. (Academic, San Diego, Calif., 1991), pp. 1059-1077.

[5] H. Hirori, K. Yamashita, M. Nagai, and K. Tanaka, "Attenuated Total Reflection Spectroscopy in Time Domain Using Terahertz Coherent Pulses," Jpn. J. Appl. Phys., vol. 43, no. 10A, pp. L1287-L1289, 2004.

[6] D. F. Plusquellic, K. Siegrist, E. J. Heilweil, and O. Esenturk, "Applications of Terahertz Spectroscopy in Biosystems," Chem. Phys. Chem., vol. 8, pp. 2412-2431, 2007. 
[7] Y. Shi and Li Wang, "Collective vibrational spectra of $\alpha$-and $\gamma$-glycine studied by terahertz and Raman spectroscopy," J. Phys. D: Appl. Phys., vol. 38, pp. 3741-3745, 2005.

[8] C. C. Homes, G. L. Carr, R. P. S. M. Lobo, J. D. LaVeigne, and D. B. Tanner, "Silicon beam splitter for far-infrared and terahertz spectroscopy," Appl. Opt., vol. 46, pp. 7884-7888, 2007.

[9] K. K. Chittur, "FTIR/ATR for protein absorption to biomaterial surfaces," Biomaterials, vol. 19, pp. 357-369, 1998.

[10] M. N. Afsar and J. B. Hasted, "Measurements of the optical constants of liquid $\mathrm{H}_{2} 0$ and $\mathrm{D}_{2} 0$ between 6 and $450 \mathrm{~cm}^{-1}$," J. Opt. Soc. Am., vol. 67, no. 7, pp. 902-904, 1977.

[11] G. M. Chaban and R. B. Gerber, "Anharmonic vibrational spectroscopy of the glycine-water complex: Caluculations for ab initio, empirical, and hybrid quantum mechanics/ molecular mechanics potentials," J. Chem. Phys., vol. 115, no. 3, pp. 1340-1348, 2001.

\section{Introduction}

Terahertz $(\mathrm{THz})$ waves, which are in the gap between microwaves and the far infrared, have long been a terra incognita, mainly due to the lack of sources and detectors to facilitate efficient study of their properties, let alone apply these waves to solve real-world problems such as in industry and medicine. $\mathrm{THz}$ waves are defined as the electromagnetic waves ranging roughly from 0.1 to $10 \mathrm{THz}$ (wavelengths of $3 \mathrm{~mm}$ to $30 \mu \mathrm{m}$ ). The range of potential applications is likely to increase as absorption spectra (i.e., fingerprint spectra) of specific chemicals in the $\mathrm{THz}$-wave region are obtained, including those of illicit drugs, vitamins, sugars, pigments, pharmaceuticals, and agricultural chemicals $[1,2,3]$. In the THz-frequency range, water is extremely absorptive with an absorption coefficient at $1 \mathrm{THz}$ of approximately $200 \mathrm{~cm}^{-1}$, which is $10^{5}$ times higher than that in the visible region [4].

$\mathrm{THz}$ time-domain spectroscopy (THz-TDS) can give the complex refractive index of a material without Kramers-Kronig analysis, but obtaining information on a dissolved substance using THz-TDS is difficult because the short interaction length prevents water absorption. To obtain spectroscopic information for solutes, a time-domain attenuated total reflection (ATR) technique with $\mathrm{THz}$ coherent pulses (THz TD-ATR) has been researched [5]. At frequencies below $3 \mathrm{THz}$, which are easily measured using THz-TDS, a lack of information exists on the intermolecular interactions of solutes, and absorption peaks such as for crystallization have been never observed. No commercial mid-infrared spectrometer can measure below $12 \mathrm{THz}$. Consequently, spectroscopy in the range between 3 and $12 \mathrm{THz}$ has been studied using far-infrared spectrometry based on a Fourier transform infrared spectrophotometer (FTIR) [6]. No study has reported on the use of an ATR spectrometer in this frequency range. To confirm absorption peaks such as the intermolecular vibration of crystallization and to compare the spectra of solids and solutions, we measured a glycine sample using an ATR spectrom- 
eter based on FTIR.

\section{Material and methods}

Glycine is the simplest amino acid and it is soluble in water, with a solubility of $22.5 \mathrm{~g} / 100 \mathrm{ml}$ at $20^{\circ} \mathrm{C}$. Shi and Wang studied the collective vibrational spectra of crystalline glycine using THz-TDS and Raman spectroscopy [7]. We studied powdered glycine (Lot no. 906W2203; Kanto Chemical, Tokyo, Japan) and dissolved it in sterilized pure water at concentrations of 0,5 , 10,15 , and $20 \mathrm{wt} \%$. To measure the powder, we mixed it with high-density polyethylene powder (Shamrock, Petaluma, CA) and made pellets with a pellet-maker, which consisted of a vacuum die and pressurizer at room temperature, a pressure of about $1500 \mathrm{~kg} / \mathrm{cm}^{2}$ to minimize the decomposition from transient heating, and a vacuum of $0.5 \times 10^{-3}$ Torr. The pellets were $13 \mathrm{~mm}$ in diameter and weighed $200 \mathrm{mg}$.

Spectra in the $0.6-12 \mathrm{THz}$ range were obtained with FTIR using a silicon broadband beam splitter [8] and a deuterated l-alanine-doped triglycine sulfate (DLATGS) room-temperature detector [2]. A high-pressure mercury lamp, which has a higher intensity than a ceramic lamp at a below $3 \mathrm{THz}$, was used as the light source. Silicon was chosen for use as the internal reflection prism for the ATR method. When measuring the spectrum of the powder, the wave number resolution and accumulations were fixed throughout the experiment at $2 \mathrm{~cm}^{-1}$ and 100 times, respectively, while when measuring the spectrum of a liquid sample, the respective values were at $16 \mathrm{~cm}^{-1}$ and 500 times.

To obtain the spectrum of a dissolved substance (e.g., protein) using the ATR method, a subtraction method is required because liquid $\mathrm{H}_{2} \mathrm{O}$ absorbs infrared very strongly [9]. In a preliminary experiment, we confirmed the validity of subtraction for measuring our dissolved substance using a mid-IR FTIR/ATR spectrometer (Bruker Optics, Ettlingen, Germany). We subtracted the absorption spectrum of the glycine solution from that of $\mathrm{H}_{2} \mathrm{O}$ multiplied by the molar ratio of $\mathrm{H}_{2} \mathrm{O}$ to glycine in solution.

\section{Results and discussion}

An evanescent electric field $(E)$ is created on the ATR crystal whose intensity decays exponentially with the distance $(z)$ into the medium, $E=E_{0} e^{-z / d p}$, where $d_{p}$ is the penetration depth and the distance at which the evanescent wave attenuates $1 / e$ times the intensity at the surface of the ATR crystal. The depth of penetration of the evanescent field can be calculated using the following formula:

$$
d_{\mathrm{p}}=\frac{\lambda}{2 \pi n_{1} \sqrt{\left(\sin ^{2} \theta-\left(n_{2} / n_{1}\right)^{2}\right)}}
$$

where $\lambda$ is the wavelength of the incident light, $n_{1}$ is the refractive index of the ATR crystal (3.417 at $\lambda=360 \mu \mathrm{m}$ for $\mathrm{Si}), n_{2}$ is the refractive index of the sample (2.1 at $\lambda=360 \mu \mathrm{m}$ for water), and $\theta$ is the incident angle 
$\left(45^{\circ}\right)$. The penetration depth is a function of wavelength. As previously noted, knowing the refractive index of the sample is necessary to determine $d_{p}$. However, compensating for this accurately is impossible because little information exists on the optical constant in the THz region. Therefore, when we compared measured absorption spectra, we used a corrected spectrum divided by the wavelength instead of the penetration depth.

Fig. 1 shows the water absorption spectrum corrected for the wavelength. Using the values measured for water, the penetration depth at $\lambda=360 \mu \mathrm{m}$ is $78.02 \mu \mathrm{m}$. In this case, we estimated that the corrected absorption coefficient from our measurement data was $230 \mathrm{~cm}^{-1}$, which is in good agreement with a previous paper [10].

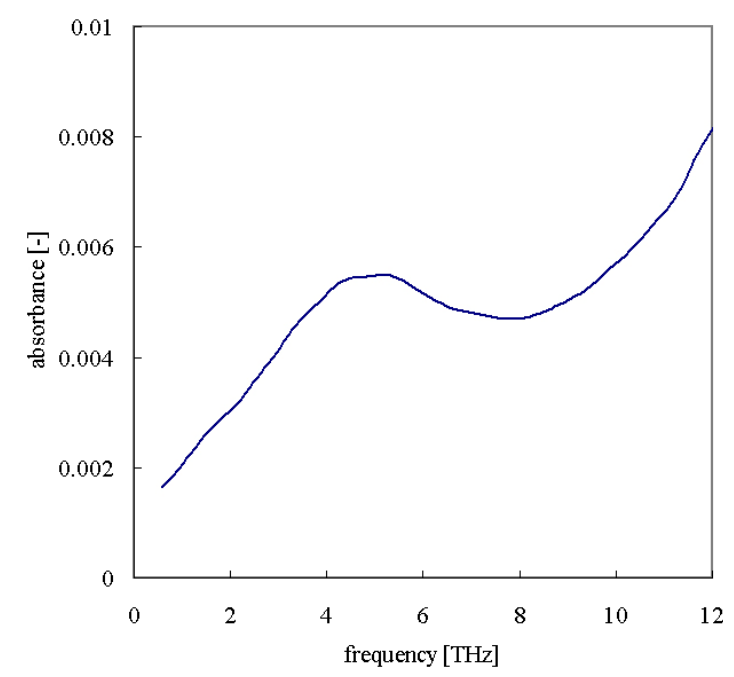

Fig. 1. Measured ATR absorption spectrum of water. In the spectrum measurement, the wave number resolution was $16 \mathrm{~cm}^{-1}$ and 500 accumulations were observed.

Fig. 2 compares the transmission and ATR spectra of glycine powder. Five absorption peaks were observed in both. When we measured the transmission spectrum using a general transmission optical system, we used pellets made from polyethylene powder mixed with glycine powder. At around 6 and $11 \mathrm{THz}$, we observed redshifts of the ATR spectrum (dashed line in Fig. 2), while no obvious redshift was seen for the small absorption band indicated by arrows in Fig. 2. This difference in the redshifts can be explained as refractive index dispersion at a large absorption band.

Fig. 3 shows the ATR absorption spectra of glycine in water. The absorption increased with the glycine concentration over the entire measurement range. In addition, the absorption peaks at 2.1, 5.2, and $9.3 \mathrm{THz}$ increased with the glycine concentration. Compared to the powder spectrum (Fig. 2), we see that the peaks at 6 and $10.7 \mathrm{THz}$ shifted to lower frequencies.

Some peaks of the glycine $-\mathrm{H}_{2} \mathrm{O}$ spectrum were redshifted from the value for pure glycine power in the mid-infrared region. Using a computational chemistry method, Chanban and Gerber reported that hydrogen bonding 


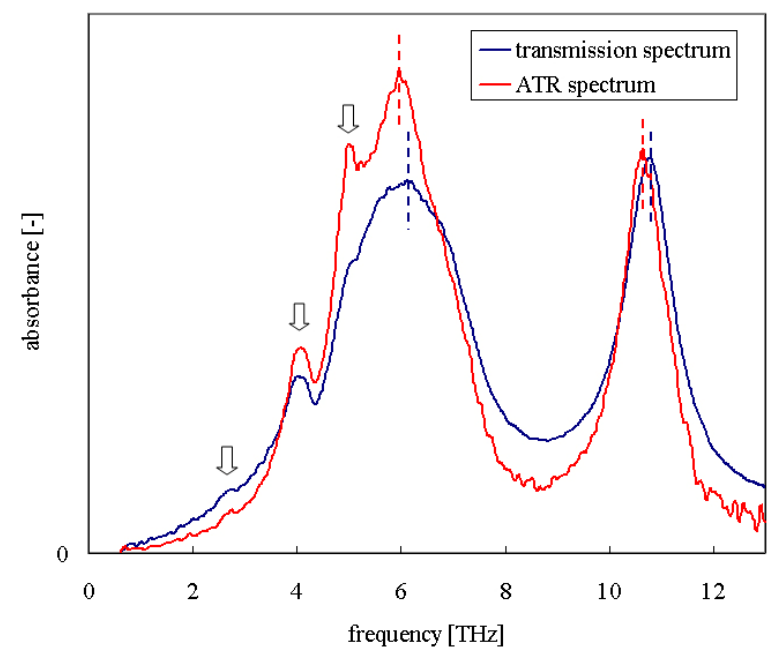

Fig. 2. Comparison of the transmission and ATR spectra of glycine powder.

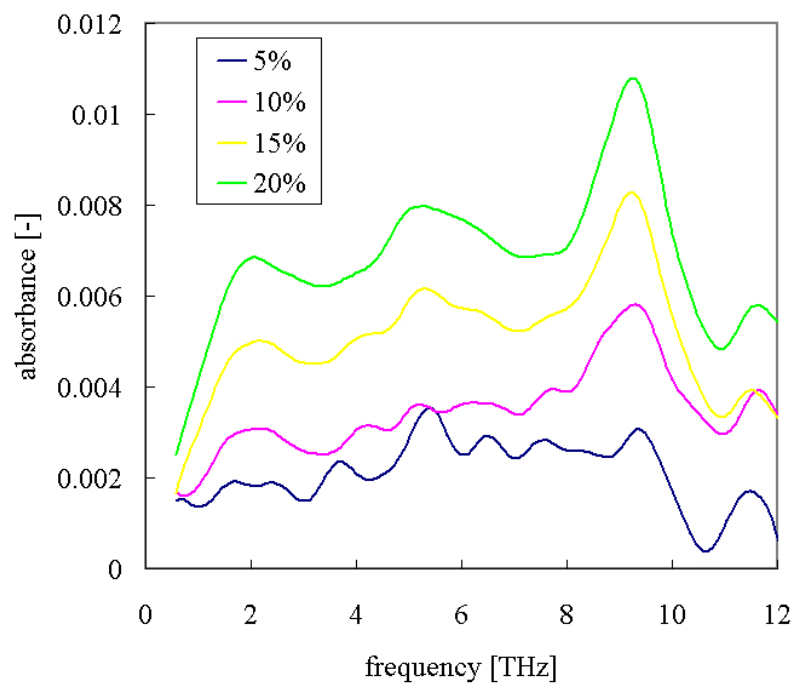

Fig. 3. ATR absorption spectra of glycine in water.

between glycine and $\mathrm{H}_{2} \mathrm{O}$ molecules produced these redshifts [11]. The $\mathrm{THz}$ region also contains spectroscopic information on hydrogen bonds, such as intermolecular bonds. We postulated that our result indicated a molecular change in the low-frequency vibrational mode of glycine due to the hydrogen bonding of glycine and $\mathrm{H}_{2} \mathrm{O}$. We anticipate determining the interaction between $\mathrm{H}_{2} \mathrm{O}$ and dissolved substances using $\mathrm{THz}$ spectroscopy. Such information can be applied to research on amino acids and sugars, and on the interactions of protein molecules with those of a solvent.

Many spectra of solids and powders in the $\mathrm{THz}$ range have been reported. Nevertheless, the origin of the absorption peaks remains unclear, which is blocking the development of spectroscopy applications. A comparison of the absorption spectra of solids and solutions should contribute to our understanding of this issue. In the future, THz-ATR spectroscopy may be extended to the inspection of food or medical supplies via, for example, mid-infrared ATR spectroscopy. 